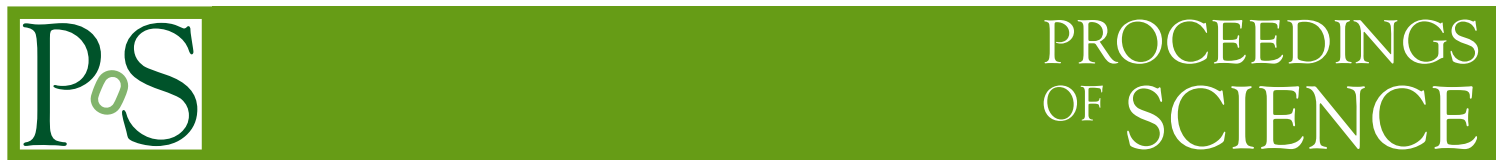

\title{
The variable sky of DAMPE
}

\author{
Qiang Yuan*, Shi-Jun Lei, and Yun-Feng Liang on behalf of the DAMPE \\ collaboration \\ Key Laboratory of Dark Matter and Space Astronomy, Purple Mountain Observatory, Chinese \\ Academy of Sciences, Nanjing 210008, P.R. China \\ E-mail: yuanqepmo.ac.cn
}

\begin{abstract}
Variable high energy gamma-ray emission reveals violent phenomena of cosmic accelerators, such as supermassive black holes, gamma-ray bursts, and probably gravitational wave bursts. The DArk Matter Particle Explorer (DAMPE) is China's first space science mission, dedicating to measuring high energy cosmic rays, electrons, and photons with high energy resolution. We report a search for variable gamma-ray emission with nearly 1.5 year DAMPE data. Two candidate variable sources, associated with active galactic nuclei CTA 102 and 3C 454.3, are found. We also search for potential short-term clusterings of arrival time of photons, and find dozens of photon pairs, one photon triple and one quadruple.
\end{abstract}

35th International Cosmic Ray Conference - ICRC2017

10-20 July, 2017

Bexco, Busan, Korea

\footnotetext{
* Speaker.
} 


\section{Introduction}

The $\gamma$-ray emission in the sky is not steady, especially for extragalactic sources. The variabilities reveal activities of accretion states of black holes, explosive phenomena of massive stars, or coalescence of compact objects. Well established variable $\gamma$-ray sources include active galactic nuclei (AGN) which host supermassive black holes, X-ray binaries which host stellar mass black holes, and $\gamma$-ray bursts (GRBs) [1,2]. Although unexpected, variabilities from the pulsar $\mathrm{J} 2021+4026[3]$ and the Crab pulsar wind nebula $[4,5]$ were also reported.

The DArk Matter Particle Explorer (DAMPE) is a satellite-borne detector for high energy cosmic rays, electrons, and $\gamma$-rays $[6,7]$. It was launched on December 17th, 2015, and has been operated for nearly 1.5 years. Here we report the preliminary results of the variable $\gamma$-ray observations with DAMPE.

\section{Gamma-ray observations of DAMPE}

The DAMPE instrument consists of four sub-detectors: a Plastic Scintillator strip Detector (PSD), a Silicon-Tungsten tracKer-converter (STK), a BGO imaging calorimeter, and a NeUtron Detector (NUD) from top to bottom [7]. For $\gamma$-ray observations, the STK measures the tracks of converted events, and the BGO calorimeter measures their energies. The PSD is used as a veto detector for charged particles. Furthermore, the BGO image of deposit energy is used to discriminate the hadronic and electromagnetic showers, in order to suppress the cosmic ray background. For more details about the photon observations with DAMPE please refer to Ref. [8].

\section{Search for variabilities}

The data analyzed in this work are from January 1st, 2016 to May 31st, 2017. The events with energies higher than $2 \mathrm{GeV}$ are selected. The instrument response functions, including the effective area and exposure time, can be found in Ref. [9].

\subsection{Long-term variabilities}

We define variabilities with time-scales from weeks to years as long-term variabilities. DAMPE covers the full sky each half year. Although the exposure is non-uniform, we have nearly three full coverages of the sky for 1.5 years of observations. We then carry out a bin-by-bin analysis to search for variabilities for each bin in the sky, with a solid angle of $\sim 3.05 \times 10^{-4} \mathrm{sr}^{\mathrm{each}^{1}}$. A weekly light curve is constructed for each bin. The left panel of Figure 1 shows an illustration of the light curve for the direction of CTA 102 , with $\left(\mathrm{RA}=338^{\circ} .2, \mathrm{Dec}=11^{\circ} .7\right)$. Shaded regions mean that the source is out of the field-of-view of DAMPE. For the time bins with zero detected event, a $90 \%$ upper limit of 2.3 expected events is derived, which corresponds to a Poisson probability of $10 \%$ when finding more than one events. For successive time bins with no events detected, we bin them together to give one upper limit. The upper limits of counts are then converted to flux upper limits through dividing by the exposure, as shown by the open triangles.

\footnotetext{
${ }^{1}$ We have applied the Mollweide projection of the sky to make sure the solid angle of every bin is identical.
} 

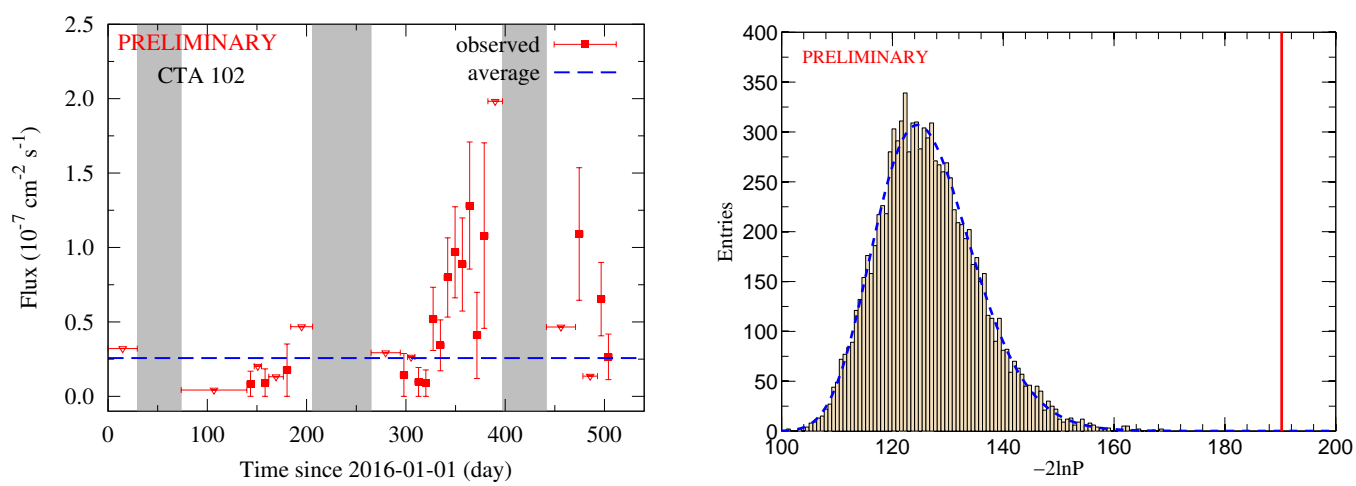

Figure 1: Left: Light curve for the direction of CTA 102. Red filled squares show the observed fluxes, open triangles represent the $90 \%$ upper limits, and the blue line represents the average flux in the whole time range. Right: Distribution of $-2 \ln P$ for $10^{4}$ simulations assuming Possion process for a steady source, for the direction of CTA 102 . The blue dashed line shows a fitting curve of a $\chi^{2}$ distribution, and the red vertical line marks the result for the DAMPE data.

The blue dashed line in the left panel of Figure 1 shows the average flux of the source during the 17 months of time. Note that this does not represent the quiescent flux of the source, since it is too low to be detected by DAMPE. Compared with the average flux level, a clear flaring activity of CTA 102 can be seen in November to December of 2016 [10].

To quantify the variability of each pixel in the sky, we construct a probability to describe the deviation of observed event numbers from expectation assuming steady emission

$$
P=\prod_{i=1}^{N_{\text {bin }}} \frac{\lambda_{i}^{k_{i}} e^{-\lambda_{i}}}{k_{i} !}
$$

where $\lambda_{i}\left(k_{i}\right)$ is the expected (observed) number of counts in the $i$ th time bin. To assess how variable the photons from one pixel are, we run toy Monte Carlo simulations of photon arrival time assuming the emission is steady, and calculate the probability of each realization. The right panel of Figure 1 shows the distribution of $-2 \ln P$ for $10^{4}$ simulations. After subtracting a constant value of $\sim 86$, the distribution of $-2 \ln P$ can be well-fitted by a $\chi^{2}$ distribution with a degree-of-freedom of $\sim 40$, as shown by the blue dashed line. The red line shows the result for the DAMPE data. From the fitting distribution we find that there is only about $1.3 \times 10^{-7}$ probability that the realization gives a $-2 \ln P$ value larger than the observed one. It suggests that the emission from this pixel is variable at $5.3 \sigma$ confidence level.

We repeat this process for the all-sky data, and found that two directions show variabilities for a confidence level $\gtrsim 99.99 \%$. One is coincident with the direction of CTA 102, and the other is coincident with the direction of 3C 454.3. Both sources are AGNs with activities confirmed by other detectors $[11,12,13,14]$. We show in Figure 2 the light curve (left) and $\ln P$ distribution for the direction of 3C 454.3. A strong flare can be seen from 3C 454.3 around June 22nd, 2016, consistent with that observed by AGILE [13] and Fermi-LAT [14].

\subsection{Short-term variabilities}

GRB-like events may show short-term variabilities (e.g., seconds to minutes) which are not 

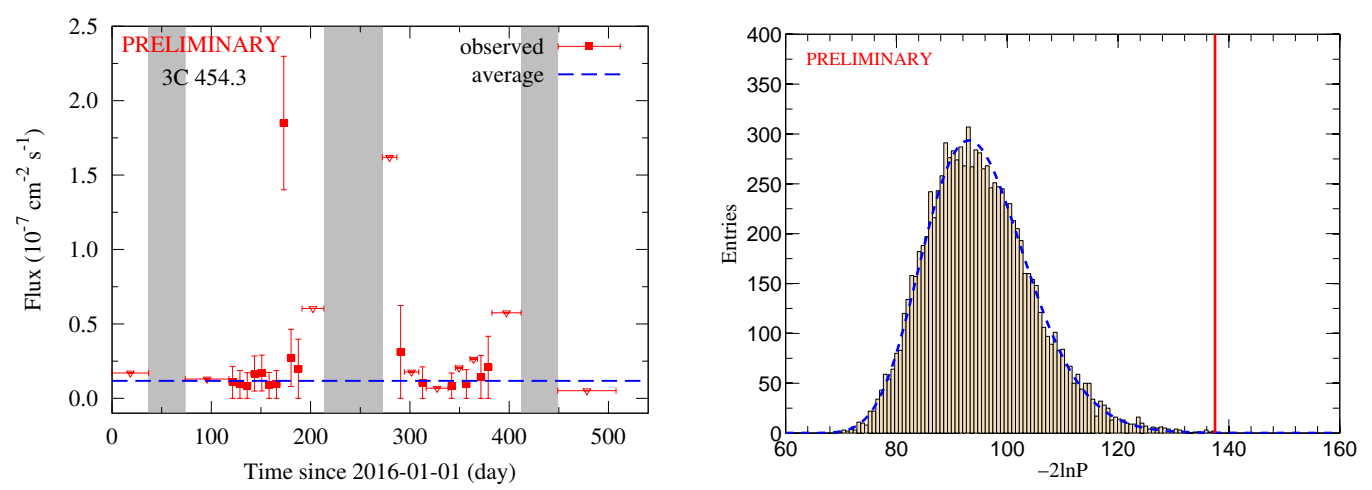

Figure 2: The light curve (left) and $-2 \ln P$ distribution (right) for the direction of $3 \mathrm{C} 454.3$.

able to be probed with the previous method. We search for such kind of events through simply searching for photon pairs or clusterings within specified time window and angular distance. Some of Fermi-LAT GRBs show GeV emission lasting to $10^{3} \mathrm{~s}$ after the trigger [15]. In addition, a pixel in the sky could be visible by DAMPE for a time as long as $\sim 1500 \mathrm{~s}$ in one orbit, given an incident angle of $50^{\circ}$ [8]. Therefore we set a time window of $1500 \mathrm{~s}$ for the search for clusterings. We further require that the angular distance $\Delta \theta$ between the photons is smaller than 1 degree, according to the angular resolution of DAMPE [7]. For clusterings of more than two photons, we require that the time difference between successive photons is smaller than $\Delta t$ and the angular distance of every two photons is smaller than $\Delta \theta$. We find in total 63 photon pairs, one photon triple, and one quadruple in the DAMPE data. Figure 3 shows the coordinates of identified photon clusterings on the DAMPE $\gamma$-ray sky. We find that some of the photon clusterings coincide with locations of known AGNs, such as CTA 102, 3C 454.3, Mkn 421, PKS 2023-07, and BL Lacertae.

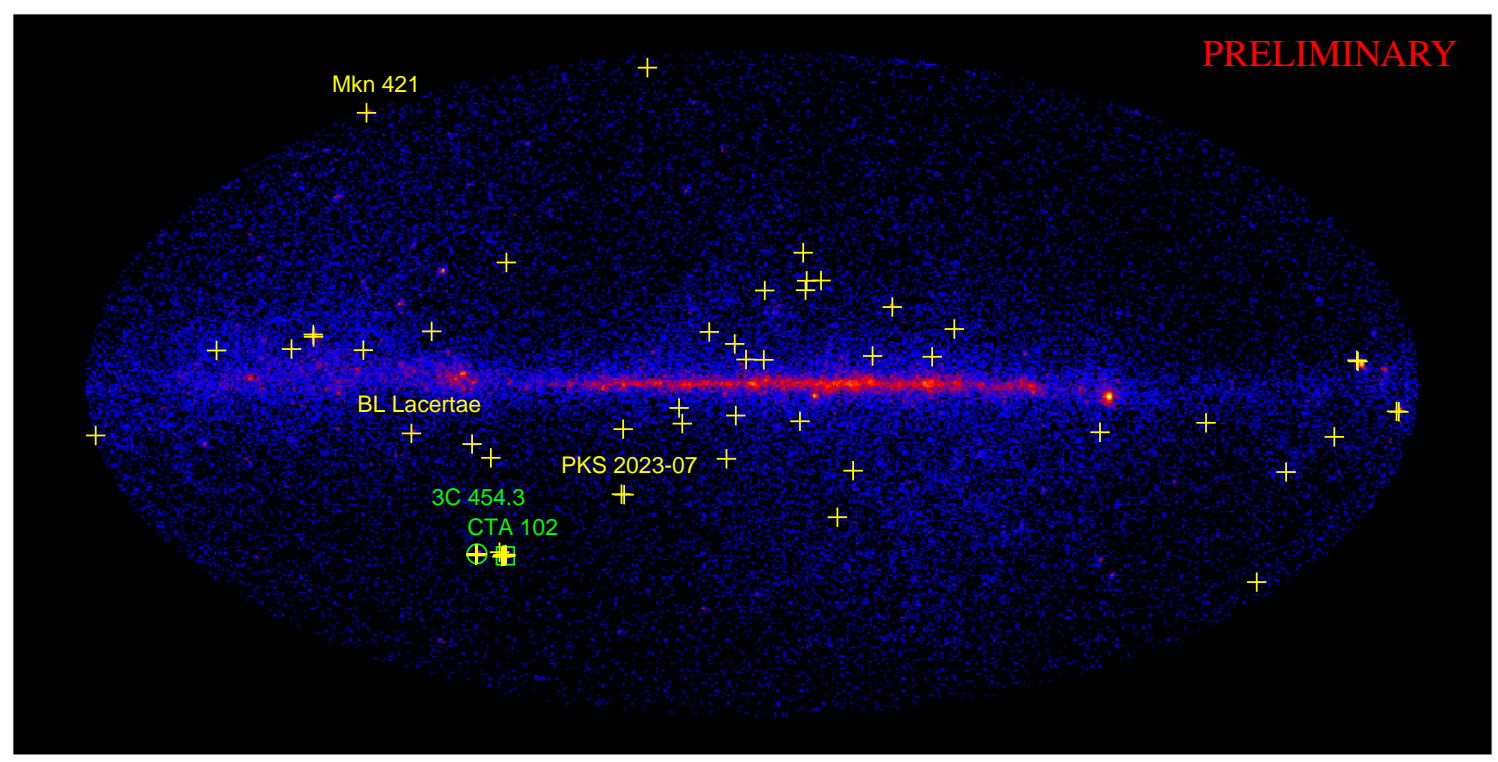

Figure 3: Skymap of DAMPE photon counts overlaid with the coordinates of photon clusterings. Crosses are for photon pairs, the circle is for the triple, and the box is for the quadruple. 


\section{Summary}

In summary, we study variable emission of $\gamma$-rays recorded by DAMPE. The long term variabilities are investigated via a comparison of weekly light curve of each pixel of the sky with that expected assuming steady emission. Two AGNs, CTA 102 and 3C 454.3, are found to be significantly variable at time scales longer than one week. We also search for photon clusterings which may potentially come from burst-like events such as GRBs. We find 63 photon pairs, one photon triple, and one quadruple which satisfy the criteria of $\Delta t<1500 \mathrm{~s}$ and $\Delta \theta<1^{\circ}$.

\section{Acknowledgements}

The DAMPE mission is funded by the strategic priority science and technology projects in space science of Chinese Academy of Sciences. The analysis of this work is supported in part by the National Key Research and Development Program of China (No. 2016YFA0400200), National Natural Science Foundation of China (No. U1631111) and the 100 Talents Program of Chinese Academy of Sciences.

\section{References}

[1] M. Ackermann et al. [Fermi-LAT Collaboration], The Fermi All-sky Variability Analysis: A list of flaring gamma-ray sources and the search for transients in our Galaxy, Astrophys. J. 771 (2013) 57 [arXiv:1304.6082].

[2] S. Abdollahi et al., The second catalog of flaring gamma-ray sources from the Fermi All-sky Variability Analysis, arXiv:1612.03165

[3] A. Allafort et al. [Fermi-LAT Collaboration], PSR J2021+4026 in the Gamma Cygni Region: The First Variable Gamma-Ray Pulsar Seen by the Fermi LAT, Astrophys. J. 777 (2013) L2 [arXiv:1308.0358].

[4] M. Tavani et al. [AGILE Collaboration], Discovery of Powerful Gamma-Ray Flares from the Crab Nebula, Science 331 (2011) 736 [arXiv:1101.2311].

[5] A. Abdo et al. [Fermi-LAT Collaboration], Gamma-ray flares from the Crab Nebula, Science 331 (2011) 739 [arXiv:1011.3855].

[6] J. Chang, Dark Matter Particle Explorer: the First Chinese Cosmic Ray and Hard Gamma-ray Detector in Space, Chin. J. Space Sci. 34 (2014) 550

[7] J. Chang et al. [DAMPE Collaboration], The Dark Matter Particle Explorer mission, Astropart. Phys. submitted (2017) [arXiv:1706.08453].

[8] Z. L. Xu et al. [DAMPE Collaboration], Gamma-ray selection of DAMPE, Proc. 35th ICRC (2017).

[9] K. K. Duan et al. [DAMPE Collaboration], The performance of DAMPE for $\gamma$-ray detection, Proc. 35th ICRC (2017).

[10] Z. L. Xu et al. [DAMPE Collaboration], DAMPE detection of variable GeV gamma-ray emission from blazar CTA 102, The Astronomer's Telegram, No. 9901 (2016).

[11] G. Minervini et al., AGILE detection of enhanced gamma-ray emission from the FSRQ CTA 102, The Astronomer's Telegram, No. 9743 (2016). 
[12] S. Ciprini [Fermi-LAT Collaboration], Fermi LAT observation of renewed and strong GeV gamma-ray activity from blazar CTA 102, The Astronomer's Telegram, No. 9869 (2016).

[13] P. Munar-Adrover et al., A gamma-ray flare from 3C 454.3 detected by AGILE, The Astronomer's Telegram, No. 9186 (2016).

[14] R. Ojha [Fermi-LAT Collaboration], Fermi LAT detection of strong gamma-ray activity from the FSRQ 3C 454.3, The Astronomer's Telegram, No. 9190 (2016).

[15] A. A. Abdo et al., Fermi Observations of GRB 090902B: A Distinct Spectral Component in the Prompt and Delayed Emission, Astrophys. J. 706 (2009) L138 [arXiv:0909.2470]. 